\title{
An Exploration on Connecting Industry Clusters with Educational Professional Group
}

\author{
Jie Zhu \\ Fuzhou University of International Studies and Trade \\ Fujian, P. R. China
}

\begin{abstract}
Many universities in China has adopted into application-oriented university transformation since the Chinese Ministry of Education promoted the policy of encouraging the reform between university teaching and industry production in 2015. Therefore, the influencing elements and reform effects are becoming the hot topics. The purpose of this research is to research and evaluate the effectiveness of this policy. In terms of research method, the coupling symbiosis theory in ecosystem is selected as intermediate variable, the government policy, industry participation and university adjustment are selected as independent variables, and the effectiveness and value chain influence are selected as dependent variables. The expected contribution of this research is to investigate the relationship between each variable and find out the key points in this transformation by sampling 647 universities and provide examples and suggestions to related groups.
\end{abstract}

Keywords-Educational Professional Group; Industry Cluster; Collaboration University with Industry

\section{The RESEARCH Motivations AND PURPOSES}

The development of an industry is relying on the talents, and the talents are needed to be educated by universities. There is a gap between the graduates that cultivated by schools and the talents that the industry needed. According to the survey on the 8777 college graduates by the China Youth News (2006), it showed that 51.5 percent of people do not think they had learn some useful knowledge in universities, and 39.2 percent of students could not find a job although they had 4 years study in school after they graduated. The Reporters on the Local Universities' Transformation Development Practice and Policy Research (2013) indicate that the primary employment rates of our national college graduates in 2011 are 77.8 percent. The gap on the graduates to join the industry professional career is the first motivation.

Kang and Wang (2014) pointed out that the goals for university is to educating talents, inheriting knowledge and creating technology. The orient of innovate is industries, it provided direction and financial supports on the innovation. The corporate innovate between colleges and industries are helpful to pushing both of them to developing. It is also the core to building up the regional creative system which is fit to the requirements on the regional economy and society developments. Therefore, how to make a correct recognizance in college-industries corporation innovation characteristics, in order to establish the competitive collegesindustries corporation innovated system, push the development on the university and industry, make a huge influence, becomes the most urgent problems that are needed to solve in researching and practicing in the college-industry corporation. The transformation situation in the industryuniversity interaction is the second motivation.

Considering to the current situation, Ministry of Education published the guide suggestions on leading some local common undergraduate university transformation, which is applied in 16 November, 2015. It required universities to promote revolution, including joint industry chains, professional system and experimental train and practice suggestions. It can rely on the industry-university fuse and the corporation between the school and enterprise, cultivating the applied and technical talents, strengthening students' employability and entrepreneurial abilities and serving for local economy and social development. The transformation situation about the policy-industry theory interaction is the third motivation.

Based on the above description, the research purposes of this research are showing as follows:

- to discuss industry-university interactive coupling symbiosis theory and application;

- to explore the influence of the government policies, industrial participation, university adjustment on university that resulted by coupling symbiosis theory;

- to investigate the influence of coupling symbiosis theory to the transformed universities.

\section{LITERATURE REVIEW}

This research is based on the Guidelines on Applicationoriented University Transformation in Some Local Universities which is issued by Chinese Ministry of Education, National Development and Reform Commission and Ministry of Finance in 2015. According to the local economic development planning and problems in developing local universities and colleges, the research theories and experiences from other developed universities are chosen to analyze the effectiveness of the transformation, promote the new mechanism in university co-operation, open schooling and find the core value of university, achievement of students and new service to society. 
The current literature is mainly focus on the industry transformation; economy restructure and social transformation; however, the research and literature in education transformation are rare. Review the past 4 years literature, the topics on application-oriented university transformation were summarized in 5 areas: (1) research on connection between specialty cluster and industry cluster (Sun., Ma., Xing., Song., 2011; Li., Xu., Sun., 2012; Sun.,2014; Li., 2015); (2) research on building specialty cluster by coordination pattern (Kou., 2012; Liu., 2013; Ma., Zhu., Liao., Qian., Yuan., 2015); (3) research on route, mechanism and model of small medium enterprise transformation (Chen., 2012); (4) research on specialty cluster construction between universities (Nie., 2015; Zheng., 2012); (5) research on background, reason and thinking of specialty cluster construction (Zhang., Ma., 2015).

Although the current research has found many achievements, some deficiencies still exist. Current literature are lacking of theory basis and development routes, successful reasons, difficulties were not discussed. Many of the literature are staying in describing the facts but with little analysis on relationship between the different elements. Therefore, the coupling symbiosis theory (Kang, Wang, 2014) pointed out the possibility of coupling research on university-industry creative co-operation. The coupling symbiosis theory treats the economic activities and ecoenvironment as an organic whole. Only by combining the characteristics of ecosystem and applying the coupling symbiosis theory into management is it possible to hold the direction of industrial ecology and realize the sustainable development of combination between specialty and industry.

\section{RESEARCH METHOD}

The university transformation is important for business. Eliminating the gap among industry operation, academic research and government is an immediate topic to solve. The variables are selected as follows:

Independent variables:

- Government policy: the responsibility of government interaction with industry and university is based on the policies or even the monetary fund to form, guide and promote the direction and interaction principles of industry-university research.

- Industry participation: the function of local industry and university' $s$ interaction is to connect the industrial supporting process with universities, such as internship cooperation, human resources management, employee training, management consulting, research design and creative value.

- University adjustment: the purpose of applicationoriented university is to service the local industry and organize the suitable textbook, teaching team, application teaching, student learning position, employment and creative entrepreneurial sprites.
Intermediate variables: The core principle of this research is the guidelines from Chinese Ministry of Education and the theoretical proposal of coupling symbiosis theory.

Dependent variables: The influence of coupling symbiosis theory on the interaction between university specialty cluster and local industry cluster.

\section{A. Research Method}

The qualitative analysis and quantitative analysis are used in this research. Based on the combination of theoretical research and case research, idea approving and case transforming, the possibility of this research is ensured. The quantitative analysis includes statistic summary, factor analysis, correlation analysis, variable analysis, regression analysis which is to exam the relationship between each variables and hypothesis.

Research sampling: the research sample is the universities established after 1999, 647 in totals and takes about $55.3 \%$ of the total universities in China. The interviewees are the teachers and students in universities and related people in industry clusters.

Research design: design the research proposal and hypothesis, questionnaire and scale design, data test and adjust field research.

Data collection and analysis: statistic summary and data analysis by SPSS software. The analysis focuses on the factors which will influence the university specialty cluster transformation for application-oriented university and industry. Then it is also important to provide the solutions as the evidence for university transformation.

\section{B. Research Contents}

The status analysis of undergraduate universities transforms to application-oriented in order to promote specialty cluster construction. This study subjects from the regional economic characteristics of industry to find the demand for talents analysis, university specialty cluster, cross-courses of professional group construction. The current situation analysis is focus on the industrial clusters to some professional group. And then, an evaluation system of the professional group and the industrial group is established. So that it can be compared and analyze each cluster docking results, the key success factors and difficult points of construction process. What' s more, the evaluation system will be used as the basis for the further study of undergraduate universities in build a professional specialty group route.

The mechanism analysis of undergraduate universities transforms to application-oriented in order to promote the connection between university specialty cluster and industry cluster. This study analyzes the construction of undergraduate universities specialty cluster by answering the questions about how to link up with industrial group? How to make analysis and evaluation? What are the influencing factors? What are the key success factors and difficult points of the construction process? Reviewing from the existing research, the mechanism analysis of undergraduate 
universities translate to application-oriented in order to promote the connection between university specialty cluster and industry cluster is still in the beginning stages. This study will base on the previous researches, from the whole process of undergraduate universities to analyze the different clusters in both universities and industry. Talking the mechanism of cluster construction is the way to clear the realization route. It is important to start from the local regional economic, set a system to make use of the undergraduate universities courses to promote industrial cluster development and shorten the gap of study and application.

The case study of undergraduate universities transforms to application-oriented in order to promote specialty cluster construction. The application-oriented university construction is his basic theory to service the local industry. Combining with an instance of transformation pilot program of undergraduate universities in our country, the analysis is deep into the standards of undergraduate universities specialty cluster, the successful experience of professional university-industry connection and the possibility and necessary of this transformation.

\section{Research Breakthrough and Emphasis}

- To provide coupling interactive model and value chain for university application-oriented transformation, there is a complementary relationship between universities and local industry. During the process of interaction and collaborative innovation, the new creative value chain is created. The theory of value chain is put forward by Michael porter which is intended to describe the different function of various interior enterprise businesses. It is also forming the evolution of an enterprise by multiple enterprises together, creating a new kind of industrial interaction model and industrial coupling interaction model.

- The influence mechanism on industrial clusters and specialty cluster connection: the establishment and development of professional university specialty cluster setting is influenced by two factors. The first one is the internal driving force of the higher education's own development needs. The second is the external driving force of the demand of industrial economic environment. What are the internal and external factors affecting the university specialty cluster construction to connect industry group. It is important to find the industrial optimization rule and relationship between specialty cluster and integrating professional courses, construct the intercollegiate systemic professional clusters for industrial docking, and build the university to university specialty cluster and innovative concept.

\section{EXPECTED OUTCOMES}

\section{A. Practical Application Value}

1) Provide the proposal for university applicationoriented transformation. According to the changes in demand and supply of technology application resulted by the Chinese economic development, the changes in talent demand, industry restructure, new technology update, occupation are changed. The transformation should be taken place in university-running system, specialty construction, teaching model, talent training, teaching team building and management service model. In terms of value chain, except the core function of university education and local industry, it is important to find the creative add-value and new cooperation.

2) Application guidelines for combination of university specialty cluster and industry cluster.

3) There is a gap between the students' learning and employment after graduation. The knowledge learned is hard to use in the real business operation. Therefore, the influencing factors of the transformation are analyzed in this research. The interacted mechanism of application transformation and local industry is shown. The specific solutions will be suggested.

\section{B. Academic Theoretical Significance}

1) Introduction system coupling theory and method: MBAlib (2015) industrial ecosystem-couple is a physics concept that a phenomenon through various interactions to influence each other among two or more systems. Economics management theory research and analysis of the coupling principle to search the best way of combination among two or more industry interact each other to be mutual promotion, mutual support, coordinated development.

2) To achieve scientific management of industrial ecosystem: Karaomerioglu (1999) had proposed the fundamental of industrial coupling interaction model is based on the indirect production process due to the division of labor. It takes the combination of theory research and evidence analyze, promote college-production creative cooperation and college-production creative level, pull the development of regional economic society, is a huge pressure and historical occasion benefit to industrial economics and promotion of college education and development of creative.

3) Developing college education industrial and regional economic industry interact research on the theory of transformation and upgrading: Li Jianghong, Lv Zheng (2006) and so forth think that industrial coupling interaction model is a dynamic interaction process. From the interaction process, industrial boundary close connection and cross overlap. On the upstream link on the value chain the outsourcing services provided, on the middle reaches of the mutual penetration, on the downstream links to provide logistics, marketing and after-sale service.

\section{DISCUSSION AND CONCLUSIONS}

This research will provide a new example and vision for the application-oriented university transformation. However, the implemented proposal and successful factors and difficulties have not formed appropriately. In order to solve 
this problem, it is important to clear the relationship between university specialty cluster and industry cluster or other related variables.

Difficulties: the function of market can promote the small medium enterprise to transform, however, many of these companies are difficult to update. The universities do not provide relevant courses for related industry cluster, therefore, the gap of learning and practice exists. The unbalance of talent demand and supply is difficult for industry cluster construction. There is no differentiation between different universities and the specialty resource sharing is very low. The specialty cluster construction is usually combined inside the university for relevant subjects. It is necessary to break the boundaries of different universities to enhance the scale and the constellation effect.

According to the Book of Changes chapter 12 in the fourth section, if you do things do not beyond the form; it is referred to as the "Tao". If you do thing beyond the form, it is called a "Unit". If you understand the meaning of different things and learn the good, it is called "Change". If you can implement the good things you have learned, it is called "Tong" . If you can teach these good things to the people in the country, it is the cause. Mr. Nan Huaijin said, the real cause is for the benefits of all the people, otherwise, it is your own career. Therefore, the university education is a cause, a cause for the benefits of teachers, students and industry, because the transformation of application-oriented university is to find the "Tao" and change it into "Tong" by connecting the specialty cluster and industry positions. This is the benefits for teachers teaching and research, student's career and employability and innovated course book.

\section{REFERENCE}

[1] P. Kang., W. Wang. Coupling symbiosis research for universityindustry creative co-operation. Journal of Technology Economics and Management. Vol. 5:17-19. 2014.

[2] Z. Ma., Y. Zhu., Y. Liao., L. Qian., B. Yuan. Research on specialty construction model for local new established universities. Journal of Second Normal College. 28(1):10-12. 2015.

[3] F. Sun. Research on specialty cluster settings for colleges form the Collaborative University-industry Perspective. Higher Education Research. 35(7): 45-48. 2014.

[4] D. Sun., X. Ma., Y. Xing., Y. Song. The Research on specialty cluster construction problems. Journal of Liaoning Economic Managent College. Vol. 2: 7-9. 2011.

[5] Z. Li. The research on connection of college specialty cluster and area industry cluster. Xiang Chao. 454(4): 29-32. 2015.

[6] J. Yang. 2013. Research on talent training model for Chinese college education. Journal of Fujian Normal University. 31(4): 34-36.

[7] X. Zhang., Z. Ma. The background, reason and idea of specialty cluster construction for new local established colleges. Journal of Chongqing Second Normal College. 28(3):22. 2015.

[8] Y. Chen. Research on entrepreneurial talent training model for Chinese college education. Journal of Ocean University of China. Vol. 10: 45-47. 2012.

[9] Z. Zheng. The thoughts about college specialty cluster construction. Journal of Henan Education College. 31(4):56-57. 2012.

[10] J. Huang. The investigation on apprenticeship talent training model for secondary vocational schools. Journal of Szechwan University. Vol. 12: 34-36. 2014. 